\title{
Subjective Daily Physical Activity Measures in Heart Disease: A Systematic Review
}

\author{
Michael J. Shoemaker, Michaela Mattern, Hannah Scholten, Jessica Zeitler, and Shweta Gore
}

\begin{abstract}
Background: The measurement of daily physical activity (DPA) is important for the prognosis and quantifying clinical outcomes in individuals with heart disease. The measurement of DPA is more feasible using subjective measures when compared with objective measures. The purpose of this systematic review of the literature was to identify the subjective measures of DPA that have established reliability and validity in individuals with heart disease to assist clinician and researcher instrument selection. Methods: A systematic search of PubMed, CINAHL Complete, PsycInfo, and Web of Science Core Collection databases was performed. Methodological rigor was assessed using 3 different quality appraisal tools. Qualitative synthesis of included studies was performed. Results: Twenty-two unique studies covering 19 subjective DPA measures were ultimately included. Methodological rigor was generally fair, and validity coefficients were moderate at best. Conclusions: Only 4 subjective measures that have established test-retest reliability and that provide an estimate of energy expenditure, metabolic equivalents, or minutes of DPA were compared against accelerometry or a DPA diary in patients with heart disease: SWISS Physical Activity Questionnaire, Total Activity Measure 1 and 2, and Mobile Physical Activity Logger. Depending on the clinician or researcher needs, instrument selection would depend on the recall period and the DPA construct being measured.
\end{abstract}

Keywords: accelerometry, questionnaire, reliability, validity

Low levels of daily physical activity (DPA) in individuals with heart disease have worse clinical outcomes compared with those with higher levels of DPA, including (1) a poorer $\operatorname{prognosis}^{1-7} ;(2)$ a lower aerobic capacity ${ }^{1}$; (3) lower health-related quality of life ${ }^{8-10}$; (4) increased sympathetic nervous system activity ${ }^{11,12}$; (5) more hospitalizations ${ }^{13-16}$; (6) a higher prevalence of obesity, metabolic syndrome, and risk factors for cardiovascular disease ${ }^{17-19}$; and (7) a reduced ability to participate in activities of daily living. ${ }^{8-10}$ Given that DPA is such a strong determinant of health outcomes, the American Association of Cardiovascular and Pulmonary Rehabilitation includes an assessment of DPA as a core competency for cardiac rehabilitation/secondary prevention professionals. ${ }^{20}$

The US Department of Health and Human Services' "Physical Activity Guidelines for Americans" ${ }^{21}$ focuses mainly on exercise (ie, structured DPA) and only tangentially addresses sedentary time. However, decreasing overall duration and individual bouts of sedentary time may decrease mortality risk $^{22,23}$ and should be considered as an important clinical outcome. Reductions in sedentary time and increases in total daily energy expenditure (EE) are accomplished not just through the use of exercise (ie, structured physical activity [PA]), but through increases in all other activities of daily living (ie, unstructured PA) as well. ${ }^{24}$ The use of the term "DPA" in this manuscript refers to the total daily EE (ie, the sum of unstructured and structured PA).

With regard to the measurement of DPA, subjective and objective measures of DPA and sedentary behavior are used in clinical and research settings. ${ }^{20}$ Compared with objective measures, subjective measures may be affected by self-reporting bias or recall memory issues. ${ }^{25,26}$ However, the advantages of subjective

Shoemaker, Mattern, Scholten, and Zeitler are with the Department of Physical Therapy, Grand Valley State University, Grand Rapids, MI, USA. Gore is with the Department of Physical Therapy, MGH Institute of Health Professions, Boston, MA, USA. Shoemaker (shoemami@gvsu.edu) is corresponding author. measures include the ease and efficiency of administration in the clinical setting, a minimal patient burden, a low cost, the provision of immediate feedback, and feasibility for use in population surveillance efforts. ${ }^{27}$

Subjective measures of DPA have different emphases with regard to the PA behavior measured. Some measure overall DPA/ $\mathrm{EE}$ (ie, total of structured and unstructured DPA), and some emphasize structured PA behavior, while others measure sedentary time (ie, the inverse of total DPA). The American Heart Association recommends that subjective measures of DPA account for the various dimensions (mode, frequency, duration, and intensity of a PA) and domains (occupational, domestic, transportation, and leisure time) of PA. ${ }^{28}$

The optimal subjective measure of DPA for individuals with heart disease has not yet been established. Three prior literature reviews considered subjective measures in patients with heart disease ${ }^{29-31}$ but were limited by the methodology, focus of the review, and/or populations considered. No prior review has specifically commented on the methodological limitations of existing validity studies of subjective daily PA measures in heart disease.

The review by Le Grande et $\mathrm{al}^{29}$ was limited in several important ways. Of the 23 listed measures included in the review, only 13 had been used in patients with heart disease, and only 9 of those 13 were validity studies.

Ahlarbi et $\mathrm{al}^{30}$ conducted a narrative literature review of studies that examined both self-report and direct PA measures and restricted their review to only patients undergoing cardiac rehabilitation. They ultimately included 8 studies covering 8 subjective PA measures. Only 4 of the 8 studies measured reliability, and the validity coefficients were generally low, especially when compared with direct/objective measures of PA. ${ }^{30}$ They concluded that, although most subjective measures of PA allow for an estimation of the metabolic equivalents of task (METs) or EE, the standard MET calculations of $3.5 \mathrm{~mL}$ oxygen $\cdot \mathrm{kg}^{-1} \cdot \mathrm{min}^{-1}$ may be an overestimate in patients with heart disease. ${ }^{30,31}$ 
Panguntalan and Gregoski ${ }^{32}$ performed an integrative literature review of studies that examined self-report and direct measures of PA in African American women "in the context of the risk for [coronary heart disease]." The review included 7 studies covering 9 self-report questionnaires estimating METs, EE, or other measures of PA. The authors observed that the included studies did not exclusively or even primarily include African American women with coronary heart disease and concluded that reliability was understudied (only 3 studies) and that only modest validity coefficients were observed. ${ }^{32}$

In summary, the measurement of DPA is important for the prognosis and quantifying clinical outcomes in individuals with heart disease. The measurement of DPA is more feasible using subjective measures compared with objective measures. Previously published reviews of subjective measures of DPA in individuals with heart disease are limited by either the methodology (ie, not a systematic review) or focus on a narrow population (ie, only cardiac rehab). Therefore, the purpose of this paper was to conduct a systematic review of the literature to identify the subjective measures of DPA that have established reliability and validity in individuals with heart disease to assist clinician and researcher instrument selection. The authors hypothesize that there will be few subjective measures of DPA that have been validated against objective measures of EE in individuals with heart disease.

\section{Methods}

\section{Literature Search}

The literature search was performed using 4 different databases: PubMed, CINAHL Complete, PsycInfo, and Web of Science Core Collection. The searches were performed on July 11, 2019. The following Medical Subject Headings (MeSH) terms and keywords were used for the search strategy: $(()(()(()(()(()(()$ (")hysical activity") OR "activities of daily living") OR "motor activities") OR walking) OR exercise) AND "heart failure") OR "heart disease") OR "cardiovascular disease") AND "surveys") OR "questionnaire”) OR “interview”) OR “log”) OR “self-report”) OR “diary, health") OR “subjective") AND "validation") AND "validation studies") AND validity) AND reliability)).

\section{Article Selection}

Included articles met the following inclusion criteria: (1) written in English, (2) included subjects with heart disease, and (3) assessed the validity and/or reliability of subjective measures of DPA. Heart disease was defined as heart failure, coronary artery disease, congenital heart defects, cardiovascular disease, a history of myocardial infarction, and valvular disease. The subjective measures of DPA were defined as instruments that provided a derived measure of EE or quantified some aspect of DPA. Articles were excluded if they (1) were not peer reviewed, (2) did not include adults in the sample, or (3) did not provide the results of the subgroup analyses for the subjects with heart disease when subjects without heart disease were included in the sample. There was no limit to the year of article publication. Following removal of duplicates, article titles were reviewed, followed by a review of the abstracts and full text, if appropriate. Article selection bias was addressed using 2 authors (each article assigned to 2 of the following authors: M.M., H.S., and J.Z.) who independently completed the screening process and a third author (S.G. or M.J.S.) resolving any conflicts.

\section{Evaluation of Included Articles}

The Quality Appraisal of Reliability Studies was used to assess the quality and applicability of the diagnostic reliability studies. ${ }^{33}$ The Quality Appraisal of Reliability Studies is an 11-item appraisal tool that was developed according to epidemiologic principles, current quality assessment checklists, and the Standards for Reporting of Diagnostic Accuracy and Quality Assessment of Diagnostic Accuracy Studies guidelines. ${ }^{33}$ The methodological quality of validity studies was assessed using the Quality Appraisal tool for Validity Studies. ${ }^{34}$ The Quality Appraisal tool for Validity Studies is a 24item instrument that examines content, concurrent, and construct validity, as well as numerous principles of methodological quality. ${ }^{34}$ The Strengthening the Reporting of Observation Studies in Epidemiology Statement was used to assess the quality of reporting of observational studies. ${ }^{35}$ The Strengthening the Reporting of Observation Studies in Epidemiology Statement is a 22-item checklist that provides recommendations to authors about how to enhance the reporting of observational studies and also aides in the critical evaluation and analysis of studies by reviewers. ${ }^{35}$ Therefore, the assessment of methodological rigor using the Quality Appraisal of Reliability Studies, Quality Appraisal tool for Validity Studies, and Strengthening the Reporting of Observation Studies in Epidemiology allowed the authors to identify the risk of bias in individual studies as part of the overall synthesis of data from the included studies. Consensus on scoring was reached by 3 authors (M.M., H.S., and J.Z.), with a fourth author confirming the assigned scores (M.J.S. or S.G.)

\section{Data Extraction}

The primary data used in this review included validity and reliability metrics for the various subjective DPA measures. The characteristics of each measure were recorded, including the method of administration, number of items, length of recall, and type/quantification of DPA measured. Sample sizes, sample selection, and sample demographic information, including the diagnosis, type and severity of heart disease, age, sex, and comorbidities, when reported, were used to assist in the assessment of risk of bias across studies. The data were extracted by the authors (M.M., H.S., M.J.S., and J.Z.) working in consultation with one another to ensure accuracy.

\section{Results}

The initial search produced 3758 results, with 2351 articles remaining after the removal of duplicates (Figure 1). Two researchers independently screened all titles/abstracts. A third author adjudicated differences. The full text was independently reviewed for 42 articles by 4 authors (M.J.S., M.M., J.Z., and H.S.), who reached agreement on the inclusion of 19 articles. Fifteen articles were excluded for not meeting the inclusion criteria, one was excluded for only being an abstract, and one was excluded for not being peer reviewed. Four additional articles were screened after a review of the included articles' references. Of note, 2 separate papers by Garet et $\mathrm{al}^{36,37}$ reported on the same study/data set. Therefore, only 22 unique studies are represented in this review.

\section{Summary of Included Studies}

The demographics of the samples studied in the included articles were as follows: the age ranged from 15 to 83 years old, with both 


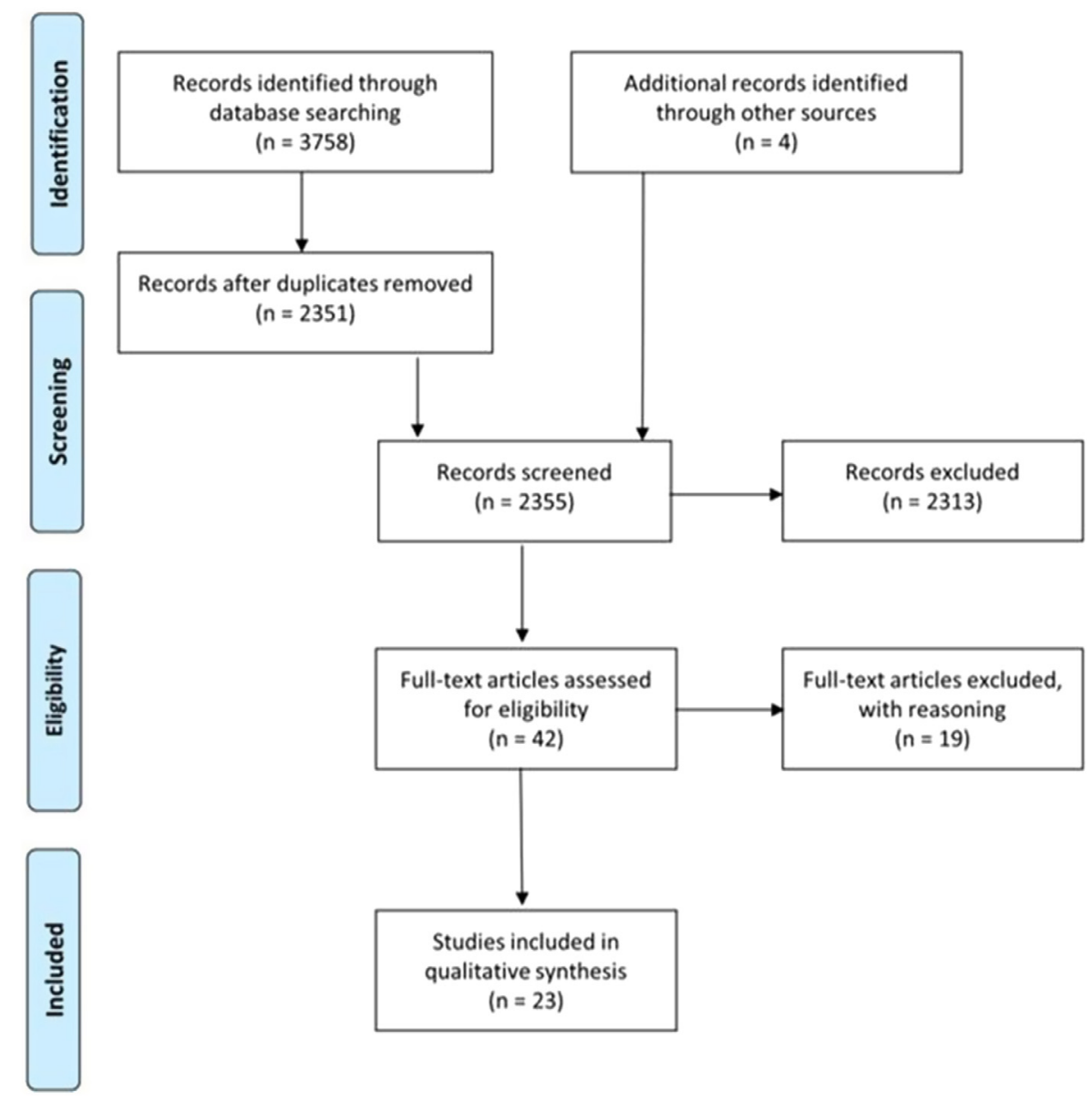

Figure 1 - PRISMA flow diagram.

men and women included (Tables 1 and 2). With regard to the diagnoses and settings studied, the most common setting was cardiac rehabilitation, with a large majority of the studies including patients with coronary artery disease (11 studies). ${ }^{38,39,41,43-45,48-52,55}$ Other diagnoses were cardiac transplant, ${ }^{53}$ cardiovascular disease, ${ }^{46}$ cardiomyopathy, ${ }^{54}$ congenital heart disease,${ }^{42,47}$ and individuals with heart failure. ${ }^{36,37,40,56-58}$

A total of 19 subjective DPA measures were studied across the 22 unique included articles. Thirteen were self-report only, ${ }^{36-44,46,51,53,55,56} 4$ were interview only, ${ }^{45,48,49,54} 1$ was administered via interview and self-report in 2 different studies, ${ }^{50,52}$ and 1 was administered via interview. ${ }^{57,58}$ The length of recall for the questionnaires ranged from $<1$-day recall to "adult life" recall, with the most common recall period being 7 days (6 instruments). ${ }^{43,44,47,56,59}$ The types of DPA that were measured included leisure-, occupational-, housework-, and transportationrelated activities. The majority of the questionnaires focused on the intensity level of DPA. The most common measurements obtained from these questionnaires were EE estimates in kilocalories or METs, minutes of moderate to vigorous PA (MVPA), and estimated peak oxygen uptake. Some questionnaires measured the frequency of exercise-induced sweating, categorized activities by intensity level, or used scale-specific scores.

All 22 unique included studies were observational studies, with 14 explicitly designed to test validity, ${ }^{36-38,41,42,44-46,50-52,54,55,58}$ 5 that tested validity as a secondary purpose, ${ }^{39,43,48,53,57}$ and 3 that reported validity coefficients but did not have an explicitly stated purpose of establishing validity. ${ }^{40,47,56}$ (Table 3). They assessed a wide variety of validity types, including convergent, concurrent, divergent, construct, and content validity. The majority of studies assessed either convergent or concurrent validity, with 13 of the included studies assessing convergent ${ }^{39,42,43,46-48,50-54,56,57}$ and 8 assessing concurrent ${ }^{36-38,41,44,45,48,49,58}$ validity.

Subjective DPA measures were compared against a variety of reference standards. The most common reference standards used to assess validity of the questionnaires were cardiopulmonary exercise testing (peak oxygen uptake [7 instruments $]^{36,37,41,47,48,53,54,57,58}$ and peak work [3 instruments] $]^{49,50,52,56)}$ and triaxial accelerometry $(8$ instruments). ${ }^{38,42-46,52}$ Other reference standards used included the 6-minute walk test (4 instruments), ${ }^{49,50,56-58}$ uniaxial accelerometers/pedometers, other subjective questionnaires, quality of life instruments, and a variety of clinical characteristics.

\section{Methodological Rigor}

The scores for methodological rigor are presented in Supplementary Materials 1, 2, and 3 (available online), respectively. Limitations in methodological rigor included a lack of specificity of the type of validity investigated (17 articles, 74\%) and not including a priori sample size calculations (17 articles, 74\%). Only 9 articles (39\%) included both the setting and time frame, and 13 articles $(56 \%)$ included either the setting or time frame. Consultation with an expert panel was mentioned in 2 articles (8\%). However, nearly all articles included a good description of the 


\begin{tabular}{|c|c|c|c|c|c|c|}
\hline Author & $\begin{array}{l}\text { Diagnosis } \\
\text { (es) }\end{array}$ & $\begin{array}{l}\text { Sample size; } \\
\text { sample location }\end{array}$ & $\begin{array}{l}\text { Gender } \\
\text { (male/ } \\
\text { female) }\end{array}$ & $\begin{array}{l}\text { BMI } \\
\text { mean (SD) }\end{array}$ & $\begin{array}{l}\text { Age, y } \\
\text { mean (SD) }\end{array}$ & $\begin{array}{l}\text { LVEF, NYHA, or other index of } \\
\text { severity }\end{array}$ \\
\hline Bahler et $\mathrm{al}^{38}$ & $\begin{array}{l}\text { MI } \\
\text { CAD }\end{array}$ & $\begin{array}{l}48 \\
\text { Switzerland }\end{array}$ & $37 / 11$ & $27.00(4.00)$ & $60.00(8.00)$ & NR \\
\hline $\begin{array}{l}\text { Tayor-Piliae } \\
\text { et } \mathrm{al}^{39}\end{array}$ & $\begin{array}{l}\text { MI } \\
\text { CAD }\end{array}$ & $\begin{array}{l}500 \\
\text { United States }\end{array}$ & $197 / 303$ & $31.50(7.20)$ & $45.90(6.40)$ & NR \\
\hline Chien et $\mathrm{al}^{40}$ & $\mathrm{HF}$ & $\begin{array}{l}111 \\
\text { Taiwan }\end{array}$ & $69 / 42$ & $25.70(4.93)$ & $63.20(11.50)$ & $\begin{array}{l}\text { LVEF: } 48.90 \%(16.40) \\
\text { NYHA: I-III }\end{array}$ \\
\hline Gruner et $\mathrm{al}^{41}$ & CAD & $\begin{array}{l}233 \\
\text { Switzerland }\end{array}$ & $186 / 47$ & NR & $63.00(10.00)$ & NR \\
\hline $\begin{array}{l}\text { Garet } \\
\text { et } \mathrm{al}^{36,37}\end{array}$ & $\mathrm{HF}$ & $\begin{array}{l}105 \\
\text { France }\end{array}$ & $87 / 18$ & $25.80(4.00)$ & $55.80(12.40)$ & $\begin{array}{l}\text { LVEF: } 33.20 \%(6.10) \\
\text { NYHA: I-IV } \\
\text { VO }_{2} \text { peak: } 17.50(4.80)\end{array}$ \\
\hline $\begin{array}{l}\text { Larsson } \\
\text { et } \mathrm{al}^{42}\end{array}$ & $\begin{array}{l}\text { Congenital heart } \\
\text { disease }\end{array}$ & $\begin{array}{l}75 \\
\text { Sweden }\end{array}$ & $46 / 29$ & $24.40(4.30)$ & $37.50(15.50)$ & $\begin{array}{l}\text { NYHA: I-III } \\
\text { Severity class: simple (39)/complex } \\
\text { (36) }\end{array}$ \\
\hline Prince et $\mathrm{al}^{43}$ & CAD & $\begin{array}{l}35 \\
\text { Canada }\end{array}$ & $19 / 16$ & $30.10(5.20)$ & $61.50(9.80)$ & $\mathrm{VO}_{2}$ peak: $19.60(6.00)$ \\
\hline Orrell et $\mathrm{al}^{44}$ & CAD & $\begin{array}{l}73 \\
\text { England }\end{array}$ & $58 / 15$ & NR & $66.23(7.74)$ & NR \\
\hline Orrell et $\mathrm{al}^{45}$ & CAD & $\begin{array}{l}72 \\
\text { England }\end{array}$ & $58 / 14$ & NR & $65.90(7.45)$ & NR \\
\hline Pfaeffli et al ${ }^{46}$ & CVD & $\begin{array}{l}30 \\
\text { New Zealand }\end{array}$ & $26 / 4$ & NR & $65.60(8.80)$ & NR \\
\hline Muller et $\mathrm{al}^{47}$ & $\begin{array}{l}\text { Congenital heart } \\
\text { disease }\end{array}$ & $\begin{array}{l}786 \\
\text { NR }\end{array}$ & $451 / 335$ & $23.70(4.00)$ & $31.10(11.60)$ & $\begin{array}{l}\text { Severity class: simple }(100) / \\
\text { moderate }(243) / \text { complex }(397) \\
\mathrm{VO}_{2} \text { peak of predicted: } 80.50(21.50)\end{array}$ \\
\hline Nowak et $\mathrm{al}^{48}$ & $\begin{array}{l}\text { MI } \\
\text { CAD }\end{array}$ & $\begin{array}{l}207 \\
\text { NR }\end{array}$ & NR & NR & $59.00(9.00)$ & $\begin{array}{l}\mathrm{VO}_{2} \text { peak: } 29.23(9.98) \\
\text { LVEF: } 51.64 \%(9.13)\end{array}$ \\
\hline Satge et $\mathrm{al}^{49}$ & $\begin{array}{l}\text { CHF } \\
\text { CAD }\end{array}$ & $\begin{array}{l}30 \\
\text { NR }\end{array}$ & $24 / 6$ & $26.20(4.00)$ & $55.00(11.00)$ & $\mathrm{LVEF}<45 \%=9(30 \%)$ \\
\hline $\begin{array}{l}\text { Gremeaux } \\
\text { et } \mathrm{al}^{50}\end{array}$ & $\mathrm{MI} / \mathrm{CAD}$ & $\begin{array}{l}52 \\
\text { France }\end{array}$ & $\begin{array}{l}\text { Non-CR: } 28 / 3 \\
\text { CR: } 31 / 5\end{array}$ & $\begin{array}{l}\text { Non-CR: } \\
27.30(4.40) \\
\text { CR: } \\
25.90(3.50)\end{array}$ & $\begin{array}{l}\text { Non-CR: } \\
58.30(11.10) \\
\text { CR: } \\
61.10(11.80)\end{array}$ & NR \\
\hline $\begin{array}{l}\text { Sundal Holen } \\
\text { et } \mathrm{al}^{51}\end{array}$ & CAD & $\begin{array}{l}217 \\
\text { Norway }\end{array}$ & $176 / 41$ & NR & $54.90(9.30)$ & NR \\
\hline $\begin{array}{l}\text { Guiraud } \\
\text { et } \mathrm{al}^{52}\end{array}$ & CAD & $\begin{array}{l}70 \\
\text { France }\end{array}$ & $58 / 12$ & $27.50(3.80)$ & $57.60(11.60)$ & NR \\
\hline Myers et $\mathrm{al}^{53}$ & Heart transplant & $\begin{array}{l}47 \\
\text { United States }\end{array}$ & $41 / 6$ & $30.00(5.40)$ & $46.70(11.60)$ & $\mathrm{VO}_{2}$ peak: $17.20(5.20)$ \\
\hline $\begin{array}{l}\text { Mezzani } \\
\text { et } \mathrm{al}^{54}\end{array}$ & Cardiomyopathy & $\begin{array}{l}\text { ALVD: } 40 \\
\text { Chronic HF: } 153 \\
\text { Italy }\end{array}$ & $\begin{array}{l}\text { ALVD: } 39 / 1 \\
\text { Chronic HF: } \\
135 / 18\end{array}$ & $\begin{array}{l}\text { ALVD: } \\
22.00(5.00) \\
\text { Chronic HF: } \\
23.00(5.00)\end{array}$ & $\begin{array}{l}\text { ALVD: } 55.00 \\
(11.00) \\
\text { Chronic HF: } \\
57.00(9.00)\end{array}$ & $\begin{array}{l}\text { NYHA ALVD: I } \\
\text { NYHA chronic HF: I-III } \\
\text { LVEF ALVD: } 29.00 \%(8.00) \\
\text { LVEF chronic HF: } 25.00 \%(8.00) \\
\text { VO }_{2} \text { peak ALVD: } 20.00(4.00) \\
\text { VO }_{2} \text { peak chronic HF: } 14.70(3.70)\end{array}$ \\
\hline Allison et $\mathrm{al}^{55}$ & $\mathrm{CR}$ & $\begin{array}{l}32 \\
\text { United States }\end{array}$ & $19 / 13$ & NR & $72.00(4.24)$ & NR \\
\hline $\begin{array}{l}\text { Borland } \\
\text { et } \mathrm{al}^{56}\end{array}$ & $\mathrm{HF}$ & $\begin{array}{l}53 \\
\text { Sweden }\end{array}$ & $\begin{array}{l}\text { Intervention: } \\
20 / 5 \\
\text { Control: } \\
18 / 5\end{array}$ & NR & $\begin{array}{l}\text { Intervention: } \\
70.00(6.00) \\
\text { Control: } \\
71.00(9.00)\end{array}$ & $\begin{array}{l}\text { Intervention group: } \\
\text { NYHA II/III: } 10 / 14(\mathrm{n}=24) \\
\text { LVEF: } 26 \%(10) \\
\text { Control group: } \\
\text { NYHA II/III: } 11 / 12 \\
\text { LVEF: } 27 \%(11)\end{array}$ \\
\hline Oka et $\mathrm{al}^{57}$ & $\mathrm{HF}$ & $\begin{array}{l}41 \\
\text { United States }\end{array}$ & $32 / 9$ & NR & $56.00(12.00)$ & $\begin{array}{l}\text { LVEF: } 23.60 \%(7.40) \\
\text { NYHA II: } 37 \% \\
\text { NYHA III: } 63 \% \\
\text { VO }_{2} \text { peak: } 17.70(3.50)\end{array}$ \\
\hline $\begin{array}{l}\text { Ribeiro- } \\
\text { Samora et al }\end{array}$ & $\mathrm{HF}$ & $\begin{array}{l}62 \\
\text { Brazil }\end{array}$ & $44 / 18$ & $25.6(3.6)$ & $47.98(10.84)$ & $\begin{array}{l}\text { LVEF: } 31.80 \%(10.4) \\
\text { NYHA I: } 7(11.3) \\
\text { NYHA II: } 24(38.7) \\
\text { NYHA: } 31(50.0) \\
\end{array}$ \\
\hline
\end{tabular}

Abbreviations: $\mathrm{ALVD}=$ asymptomatic left ventricular dysfunction; $\mathrm{BMI}=$ body mass index; $\mathrm{CAD}=$ coronary artery disease; $\mathrm{CR}=$ cardiac rehabilitation; $\mathrm{HF}=$ heart failure; $\mathrm{LVEF}=$ left ventricular ejection fraction; $\mathrm{MI}=$ myocardial infarction, $\mathrm{NR}=$ not reported; $\mathrm{NYHA}=\mathrm{New}$ York Heart Association Functional $\mathrm{Class}$; $\mathrm{VO}_{2}$ peak, peak oxygen uptake. 
Table 2 Characteristics of Subjective Daily PA Measures

\begin{tabular}{|c|c|c|}
\hline Name; author & $\begin{array}{l}\text { Method of administration; } \\
\text { no. of items; length of recall }\end{array}$ & Type of PA \\
\hline SBAS; Tayor-Piliae et al $^{39}$ & $\begin{array}{l}\text { Self-report questionnaire mailed to } \\
\text { participants prior to clinic visit; } \\
2 \text { items; up to } 1-y \text { recall }\end{array}$ & $\begin{array}{l}\text { Occupational activities ranging from mostly sedentary to hard physical labor. } \\
\text { Leisure-time activity, ranging from sedentary to vigorous activity. }\end{array}$ \\
\hline $\begin{array}{l}\text { DAQIHF; Garet et } \mathrm{al}^{36,37} \text { and } \\
\text { Chien et al }{ }^{40}\end{array}$ & $\begin{array}{l}\text { Self-report; } \\
82 \text { items; } 1 \text {-wk recall }\end{array}$ & $\begin{array}{l}7 \text { dimensions, including sleeping/resting, everyday activities, housework } \\
\text { activities, leisure-time activity, occupational activities, moving about, and } \\
\text { miscellaneous activities. }\end{array}$ \\
\hline $\begin{array}{l}\text { IPAQ Long Form; Larsson et } \mathrm{al}^{42} \\
\text { and Prince et } \mathrm{al}^{43}\end{array}$ & $\begin{array}{l}\text { Self-report; } \\
27 \text { items; 7-d recall }\end{array}$ & $\begin{array}{l}\text { Light, moderate, and vigorous activities including job-related, transportation, } \\
\text { and housework/house maintenance/caring for family. Time spent sitting } \\
\text { during typical week or weekend day. }\end{array}$ \\
\hline $\begin{array}{l}\text { IPAQ Short Form; Muller et } \mathrm{al}^{47} \\
\text { and Borland et } \mathrm{al}^{56}\end{array}$ & $\begin{array}{l}\text { Self-report; } \\
7 \text { items; 7-d recall }\end{array}$ & Vigorous, moderate, or walking activities, and 1 item on sitting time. \\
\hline $\begin{array}{l}\text { Self-reported exercise-induced } \\
\text { sweating frequency; Gruner } \\
\text { et } \mathrm{al}^{41}\end{array}$ & $\begin{array}{l}\text { Self-report; } \\
\text { No information on number of } \\
\text { items; } \\
1 \text {-mo recall }\end{array}$ & $\begin{array}{l}\text { Frequency and duration of different PA, as well as frequency of exercising } \\
\text { vigorously enough to work up a sweat. }\end{array}$ \\
\hline TAM1; Orrell et $\mathrm{al}^{44}$ & $\begin{array}{l}\text { Self-report; } \\
6 \text { items; } 7 \text {-d recall }\end{array}$ & $\begin{array}{l}\text { Strenuous, moderate, and mild activities of mainly leisure- and work-related } \\
\text { options. }\end{array}$ \\
\hline TAM2; Orrell et $\mathrm{al}^{44}$ & $\begin{array}{l}\text { Self-report } \\
6 \text { items; 7-d recall }\end{array}$ & $\begin{array}{l}\text { Strenuous, moderate and mild activities of mainly leisure- and work-related } \\
\text { options. }\end{array}$ \\
\hline $\begin{array}{l}\text { DPAQ; Guiraud } \\
\text { et al } \mathrm{al}^{50}\end{array}$ & $\begin{array}{l}\text { Self-report/interview } \\
9 \text { items; no recall }\end{array}$ & Questions on daily activities, sports and leisure activities, and rest time. \\
\hline Acti'MET; Satge et al ${ }^{49}$ & $\begin{array}{l}\text { Interview; } \\
3 \text { items; immediate use }\end{array}$ & Intensity of activity, duration of activity, and weight of subject. \\
\hline $\begin{array}{l}\text { Selected questions; Sundal Holen } \\
\text { et } \mathrm{al}^{51}\end{array}$ & $\begin{array}{l}\text { Self-report; } \\
5 \text { items; "Past few weeks" recall }\end{array}$ & $\begin{array}{l}\text { Likert-type scale on frequency, intensity, and duration of exercise, as well as } \\
\text { comparison of self to others and activity level compared to last year. }\end{array}$ \\
\hline SWISSPAQ; Bahler et al ${ }^{38}$ & $\begin{array}{l}\text { Self-report; } \\
21 \text { items; 2-mo recall }\end{array}$ & Household and leisure-time activities. \\
\hline PPAQ; Myers et $\mathrm{al}^{53}$ & $\begin{array}{l}\text { Self-report; } \\
6 \text { items; year recall and "adult life", } \\
\text { recall }\end{array}$ & Walking, stairs, sports and recreational activities, and occupational activities. \\
\hline PPAQ; Nowak et al ${ }^{48}$ & $\begin{array}{l}\text { Interview; } \\
8 \text { items; } 6 \text { mo to a year recall }\end{array}$ & $\begin{array}{l}\text { Walking, stairs, sports and recreational activities, questions about fatigue, } \\
\text { tiredness, and level of exertion. }\end{array}$ \\
\hline PASE; Allison et al ${ }^{55}$ & $\begin{array}{l}\text { Self-report; } \\
32 \text { items; up to 7-d recall }\end{array}$ & Occupational, leisure, and household activities. \\
\hline HSE; Orrell et $\mathrm{al}^{45}$ & $\begin{array}{l}\text { Interview; } \\
\text { Unclear number of items; } \\
\text { 4-wk recall }\end{array}$ & $\begin{array}{l}\text { Heavy home activities (household repairs, building work, walks }>30 \text { min, and } \\
\text { sports/exercise }>15 \mathrm{~min} \text { ). Activity while at work for those who work. }\end{array}$ \\
\hline GLTEQ; Prince et al ${ }^{43}$ & $\begin{array}{l}\text { Self-report; } \\
3 \text { items; 7-d recall }\end{array}$ & Mild/light, moderate, and strenuous exercise with examples for each level. \\
\hline MobilePAL; Pfaeffli et al $^{46}$ & $\begin{array}{l}\text { Self-report; } \\
2 \text { items } 2 \text { times per day; }<1-d \text { recall }\end{array}$ & PA level during work/daytime and during leisure time/evening. \\
\hline PAS; Mezzani et al ${ }^{54}$ & $\begin{array}{l}\text { Interview; } \\
1 \text { item; 6-mo recall }\end{array}$ & Asked to describe habitual leisure time and occupational activities. \\
\hline $\begin{array}{l}\mathrm{HAP} ; \mathrm{Oka}^{57} \text { and Ribeiro-Samora } \\
\text { et } \mathrm{al}^{58}\end{array}$ & $\begin{array}{l}\text { Unclear method of administration/ } \\
\text { interview; } \\
94 \text { items on activity, } 8 \text { items on } \\
\text { dyspnea; "Current" activities }\end{array}$ & $\begin{array}{l}\text { Leisure activity, ADLs, household activities, sports/recreation, and } \\
\text { occupational activities. }\end{array}$ \\
\hline
\end{tabular}

Abbreviations: ADLs, activities of daily living; DAQIHF, Daily Activity Questionnaire in Heart Failure Scale; DPAQ, Dijon Physical Activity Questionnaire; GLTEQ, Godin-Shephard Leisure-Time Exercise Questionnaire; HAP, Human Activity Profile; HSE, Health Survey of England; IPAQ, International Physical Activity Questionnaire; MobilePAL, Mobile Phone Physical Activity Logger; PA, physical activity; PAS, Physical Activity Score; PASE, Physical Activity Scale for the Elderly; PPAQ, Abridged Paffenbarger Physical Activity Questionnaire; SBAS, Standard Brief Activity Questionnaire; SWISSPAQ, SWISS Physical Activity Questionnaire; TAM1, Total Activity Measure 1; TAM2, Total Activity Measure 2.

outcomes being validated, procedures, and rationale for use of their reference standards. Regarding methodological rigor for reliability testing, only 2 articles (8\%) mentioned the blinding of raters, and those raters were blinded to the results of the reference standards or to information not intended to be provided as part of the testing procedure. However, nearly all studies used a representative sample of subjects, with the test applied correctly and using appropriate statistics. 


\begin{tabular}{ll}
$\begin{array}{l}\text { Questionnaire; } \\
\text { author }\end{array}$ & $\begin{array}{l}\text { Questionnaire measurement } \\
\text { used for validity }\end{array}$ \\
\hline
\end{tabular}

SWISSPAQ;

Bahler et $\mathrm{al}^{38}$

Daily MET hours

TAM1 and

TAM2; Orrell et $\mathrm{al}^{44}$
TAM1/TAM2: number of minutes of PA at each activity level to yield total activity score in minutes to allow MET minutes calculation
MobilePAL Questionnaire; Pfaeffli et $\mathrm{al}^{46}$

IPAQ-LF;

Larsson et $\mathrm{al}^{42}$

Achievement of $\geq 150 \mathrm{~min} / \mathrm{wk}$ of MVPA

Ratio of total energy expenditure and resting energy expenditure during $24 \mathrm{~h}$; average daily METs
IPAQ-SF;

Borland et al ${ }^{56}$

GLTEQ and IPAQ Long Form; Prince et $\mathrm{al}^{43}$

HSE; Orrell et al ${ }^{45}$ Activity classification of days with $>30 \mathrm{~min}$ of MVPA as low (0), medium (1-5), or high $(>5)$

Weekly totals converted for both questionnaires to average minutes per day of MVPA or sitting time
Type of validity

assessed; reference standard

Concurrent;

ACTIHEART measuring heart rate and accelerometry, and PA diary

Concurrent;

RT3 measuring accelerometry

Convergent;

Actigraph GTIM measuring accelerometry and IPAQ-LF
Convergent;

ACTIHEART measuring heart rate and accelerometry

Convergent;

pedometer measuring steps per day, peak power measured by a maximal cycle ergometer test, and 6MWT

Convergent;

ActivPAL3 accelerometer

Concurrent;

RT3 measuring accelerometry
Validity coefficients

$r=.41$ with ACTIHEART $(r=.61$ without beta

blockers; $r=.33$ with beta blockers)

$r=.41$ with diary

Overestimated activity by $1.26-1.37$ average daily MET hours

Correlations between activity score (MET per minutes) and time spent in activity (in minutes) with the accelerometer:

$r=-.09$ to .27 for TAM1 total, light, moderate, and strenuous activities

$r=.02$ to .39 for TAM2 total, light, moderate, and strenuous activities

TAM1 overestimated activity by $2000-8000$ MET minutes

TAM2 overestimated activity by $2000-5000$ MET minutes

Correlations between MobilePAL and accelerometer:

$r=.45$ for PA counts per minute

$r=.45$ for average daily MET

$r=.39$ for daily minutes of activity

Overestimated activity by 0.28 average daily METs

Correlations between MobilePAL and IPAQ-LF:

$r=.49$ for IPAQ estimated MET minutes per day

$r=.48$ for IPAQ estimated minutes of PA

Correlations for the IPAQ-LF with the accelerometer:

$r=.40$ to .62

$r=.20$

IPAQ significantly overestimated proportion of subjects exceeding $\geq 150$ min of MVPA per week

Sensitivity/specificity for detecting those with $\geq 150 \mathrm{~min}$ of MVPA per week was $83 \% / 41 \%$

IPAQ sit time (in minutes) with steps per day: $r=-.18$

IPAQ sit time (in minutes) with watts: $r=-.15$

IPAQ sit time (in minutes) with 6MWT: $r=-.11$

IPAQ sit time (in minutes) with IPAQ category: $r=-.02$

IPAQ category with steps per day: $r=.54(P<.01)$

IPAQ category with watts: $r=.24$

IPAQ category with 6MWT: $r=.35(P<.05)$

Spearman correlation between sitting time and MVPA with ActivPAL3 (IPAQ $r_{\mathrm{s}}=.05$, GLTEQ $r_{\mathrm{s}}=.20$ not statistically significant)

Change in MVPA when comparing baseline to followup, measured by both GLTEQ and ActivPAL3, was statistically significant but not correlated

Change in sitting time when comparing baseline to follow-up, measured by both IPAQ and ActivPAL3, was not statistically significant and not correlated

Sensitivity/specificity, respectively

Low PA: $0.35 / 0.92$

Medium PA: 0.40/0.56

High PA: 1.00/0.76

Activity levels from HSE failed to agree with accelerometer data $(\kappa=.08,95 \% \mathrm{CI},-0.20$ to 0.36$)$ Sensitivity analysis on above data yielded similar results ( $\kappa=.04,95 \% \mathrm{CI},-0.31$ to 0.40 )

Concurrent;

6MWT, peak power measured by a maximal cycle ergometer exercise test, and IPAQ-SF Dijon PAS
Acti'MET correlations:

$r=.54 ; r^{2}=.29$ for $6 \mathrm{MWT}$

$r=.27 ; r^{2}=.07$ for max power

$r=.88 ; r^{2}=.77$ for IPAQ

$r=.39 ; r^{2}=.15$ for PAS

Abbreviations: CI, confidence interval; GLTEQ, Godin-Shephard Leisure-Time Exercise Questionnaire; HSE, Health Survey of England; IPAQ, International Physical Activity Questionnaire; IPAQ-LF, IPAQ Long Form; IPAQ-SF, IPAQ Short Form; MET, metabolic equivalents or task; MVPA, moderately vigorous physical activity; PA, physical activity; PAS, Physical Activity Score; SWISSPAQ, SWISS Physical Activity Questionnaire; TAM1, Total Activity Measure 1; TAM2, Total Activity Measure 2; 6MWT, 6-minute walk test. 


\section{Validity of Subjective DPA Measures}

This review identified 4 subjective DPA measures that estimated total daily MET minutes or EE per kilocalories that were compared against the reference standard of accelerometry (Table 3): Swiss Physical Activity Questionnaire (SWISSPAQ), ${ }^{38}$ Total Activity Measure version 1 (TAM1), ${ }^{44}$ Total Activity Measure version 2 (TAM2), ${ }^{44}$ and Mobile Phone Physical Activity Logger (MobilePAL). ${ }^{46}$ The MobilePAL demonstrated the strongest correlation values between estimated EE per kilocalories and accelerometry $(r=.39$ to .45$))^{46}$ For the instruments that measured correlation values between the estimated MET minutes per day and accelerometry, the SWISSPAQ demonstrated the highest correlation ( $r=.41$ overall, $r=.61$ for participants without beta blockers, and $r=.33$ for participants with beta blockers), ${ }^{38}$ followed by the TAM2 $(r=.02$ to .39$)$ and the TAM1 $(r=-.09$ to .27$) .{ }^{44}$ In addition, the SWISSPAQ was moderately correlated to a PA diary $(r=.41) .^{38}$

Other subjective DPA measures (Table 3) that were compared against accelerometer-based reference standards but which only estimated a narrow aspect of PA include the Godin-Shephard Leisure-Time Exercise Questionnaire (average minutes per day of moderately vigorous PA or sitting time ${ }^{43}$ ), the Health Survey of England (days with $>30$ min of moderately vigorous $\mathrm{PA}^{45}$ ), and the IPAQ (achievement of $\geq 150 \mathrm{~min} / \mathrm{wk}$ of $\mathrm{MVPA},{ }^{42}$ general PA category, and sitting time ${ }^{56}$ ). Correlations with various accelerometer-derived measures were $<.20$, except for the IPAQ PA category and steps per day $(r=.54) .{ }^{56}$

The Acti'MET estimates daily EE per kilocalories and was compared against another subjective DPA measure of EE per kilocalories. The Acti'MET demonstrated a strong correlation between daily EE per kilocalories and the IPAQ-SF estimation of $\mathrm{EE}(r=.88){ }^{49}$

Most subjective DPA measures were compared against other, non-DPA reference standards, such as peak oxygen uptake and anthropometric measures. The Physical Activity Score, ${ }^{54}$ Daily Activity Questionnaire in Heart Failure Scale, ${ }^{36,37}$ International Physical Activity Questionnaire (IPAQ) Short Form, ${ }^{47}$ Paffenbarger Physical Activity Questionnaire, ${ }^{48}$ and Abridged Paffenbarger Physical Activity Questionnaire ${ }^{53}$ estimated the MET minutes per day or daily EE per kilocalories, but were only compared against a reference standard of peak oxygen uptake. The correlation coefficients with peak oxygen uptake ranged from .30 to .71 and from .60 to .82 for anthropometric measures.

The most commonly studied subjective DPA measure was the IPAQ (Long Form [both the entire form and sitting time] and Short Form). The IPAQ was used both as a questionnaire under investigation and as a reference standard to establish the validity of another questionnaire. In studies seeking to validate the IPAQ, various IPAQ-reported and IPAQ-calculated measures were studied, including: 1) the achievement of $\geq 150$ minutes per week of MVPA, ${ }^{42}$ 2) MET minutes per week used to categorize as healthenhancing PA, ${ }^{47} 3$ ) categories of minimally active or inactive, ${ }^{56} 4$ ) PA categories of low, moderate, and high, ${ }^{56}$ and 5) sitting time (in minutes). ${ }^{43,56}$ These IPAQ-reported measures were compared with reference standards of accelerometry, ${ }^{42,43}$ peak oxygen uptake, ${ }^{47}$ health-related quality of life, ${ }^{47}$ the Medical Outcomes Study Short Form-36, ${ }^{47,56}$ pedometer, ${ }^{56}$ peak work,${ }^{56} 6$-minute walk test,${ }^{56}$ and muscle strength and endurance tests. ${ }^{56}$ When the IPAQ was used as a reference standard to establish the validity of other subjective DPA measures, it was compared with the EE per kilocalorie estimation from the MobilePAL ${ }^{46}$ and the Acti'MET. ${ }^{49}$ The highest correlation of all comparisons using the IPAQ was with the Acti'MET estimation of EE per kilocalories $(r=.88)$, as noted above. 49

With regard to the types of analyses, most studies used correlation coefficients, with few studies using other methods for measurement of agreement, such as the kappa statistic ${ }^{41,45}$ or Bland-Altman plots. ${ }^{38,44,46,58}$ Of those studies using an objective reference standard for DPA, the subjective questionnaires generally overestimated DPA based on the lower limits of agreement in Bland-Altman analyses. For example, overestimations of METs were as follows: TAM1 by 2000 to 8000 MET minutes, ${ }^{44}$ TAM2 by 2000 to 5000 MET minutes, ${ }^{44}$ SWISSPAQ by 1.26 to 1.37 MET hours, ${ }^{38}$ and the MobilePAL by 0.28 average daily METs. ${ }^{46}$

\section{Reliability of Subjective DPA Measures}

Of the 22 unique studies included in this review, only 9 examined reliability (Table 4). The questionnaires assessed for reliability include the SWISSPAQ, ${ }^{38}$ Daily Activity Questionnaire in Heart Failure Scale, ${ }^{36,37,40}$ TAM1 and TAM2, ${ }^{44}$ MobilePAL, ${ }^{46}$ Acti'MET, ${ }^{49}$ Dijon Physical Activity Score,${ }^{50}$ Physical Activity Score, ${ }^{54}$ and Physical Activity Scale for the Elderly. ${ }^{55}$ These studies examined a variety of reliability metrics, including test-retest, interrater and intrarater reliability, reproducibility with Bland-Altman plots, and day-to-day stability over a 7-day period. Of the 6 studies that assessed test-retest reliability, the test-retest intervals ranged from 8 days to greater than 6 weeks. ${ }^{36-38,40,44,54,55}$ Test-retest reliability was measured in a variety of ways, including intraclass correlation coefficients (ICCs), Pearson's correlation coefficients, and repeated-measures analysis of variance. Of the 4 instruments whose test-retest reliability was measured by ICC, the Daily Activity Questionnaire in Heart Failure Scale, and the Physical Activity Score demonstrated the highest test-retest values (ICC $=$ .99 and .97 , respectively), ${ }^{40,54}$ followed by the TAM2 and TAM1 (ICC $=.82$ and .73 , respectively). ${ }^{44}$ Of the 2 instruments whose test-retest reliability was measured using Pearson correlation coefficients, the Physical Activity Scale for the Elderly demonstrated the highest value $(r=.72), 55$ followed by the SWISSPAQ $(r=.62) .{ }^{38}$

\section{Discussion}

Given the importance of DPA in prognosis and clinical outcomes in patients with heart disease, the clinical feasibility of subjective DPA measures, and limitations of previous reviews, the primary purpose of this review was to identify reliable and accurate subjective DPA measures in individuals with heart disease to help clinicians and researchers select an appropriate instrument. The most useful subjective DPA measure is that which provides a measure of actual activity levels in terms of METs, EE per kilocalories, or minutes per week of overall activity. This review revealed that there were 10 instruments across 10 studies that estimated either EE per kilocalories (5 instruments) $36,37,40,46,48,49,53$ or METs (5 instruments). ${ }^{38,44,47,54}$ No instrument measured the total minutes of overall activity per week, though 2 measured the minutes of MVPA per week ${ }^{42,43}$ and 2 measured the sedentary time. ${ }^{43,56}$ The validation of such measures would ideally use the most relevant reference standards of calorimetry, ${ }^{59,60}$ accelerometry, ${ }^{59-61}$ or detailed DPA diaries. ${ }^{62}$ This review observed that, of the instruments measuring EE, kilocalories, or METs, none were compared against calorimetry, 4 instruments were compared against acceleromety, ${ }^{38,44,46}$ and 1 against a detailed PA diary. ${ }^{38}$ 
Table 4 Reliability Design and Statistics of Subjective Daily PA Measures

\begin{tabular}{|c|c|c|c|c|}
\hline $\begin{array}{l}\text { Questionnaire; } \\
\text { author }\end{array}$ & $\begin{array}{l}\text { Type of } \\
\text { reliability }\end{array}$ & Outcomes measured for reliability & $\begin{array}{l}\text { Test-retest } \\
\text { interval }\end{array}$ & Reliability measures \\
\hline $\begin{array}{l}\text { SWISSPAQ } \\
\text { Bahler et } \mathrm{al}^{38}\end{array}$ & $\begin{array}{l}\text { Test-retest } \\
\text { Reproducibility }\end{array}$ & Total MET hours & $14-51 \mathrm{~d}$ & $\begin{array}{l}\text { Pearson } r=.62 \\
\text { No difference between time points, } \\
\text { Bland-Altman plot }\end{array}$ \\
\hline $\begin{array}{l}\text { DAQIHF; Garet } \\
\text { et } \mathrm{al}^{36,37}\end{array}$ & $\begin{array}{l}\text { Interrater } \\
\text { Test-retest }\end{array}$ & DEE & $>6 \mathrm{wk}$ & $\begin{array}{l}\text { Interrater: no significant difference for } \\
\text { DEE using paired } t \text { test } \\
\text { Test-retest: no significant difference in } \\
\text { DEE using paired } t \text { test }\end{array}$ \\
\hline $\begin{array}{l}\text { DAQIHF; Chien } \\
\text { et } \mathrm{al}^{40}\end{array}$ & Test-retest & DEE & Within 2 wk & $\mathrm{ICC}=.99$ \\
\hline $\begin{array}{l}\text { TAM1 and } \\
\text { TAM2; Orrell } \\
\text { et } \mathrm{al}^{44}\end{array}$ & $\begin{array}{l}\text { Test-retest } \\
\text { Reproducibility }\end{array}$ & $\begin{array}{l}\text { Total activity in minutes of daily activity and } \\
\text { METS per minute represented by total activity } \\
\text { score }\end{array}$ & $\begin{array}{l}>8 \mathrm{~d} \\
\text { otherwise not } \\
\text { specified }\end{array}$ & $\begin{array}{l}\text { TAM1 total activity } \mathrm{ICC}=.73 \\
\text { TAM2 total activity } \mathrm{ICC}=.82 \\
\text { Bland-Altman plots for TAM1 and } \\
\text { TAM2 }\end{array}$ \\
\hline $\begin{array}{l}\text { MobilePAL; } \\
\text { Pfaeffli et } \mathrm{al}^{46}\end{array}$ & $\begin{array}{l}\text { Day-to-day } \\
\text { stability over 7-d } \\
\text { period }\end{array}$ & $\begin{array}{l}\text { Ratio of total energy expenditure and resting } \\
\text { energy expenditure during } 24 \mathrm{~h}\end{array}$ & 7-d period & $\begin{array}{l}\text { No significant difference with repeated- } \\
\text { measures analysis for both } 7 \mathrm{~d} \text { and any } \\
2 \mathrm{~d}\end{array}$ \\
\hline $\begin{array}{l}\text { Acti'MET; Satge } \\
\text { et al }{ }^{49}\end{array}$ & $\begin{array}{l}\text { Interrater } \\
\text { Intrarater }\end{array}$ & Weekly energy expenditure & $\begin{array}{l}\text { Interrater: } \\
\text { same day } \\
\text { Intrarater: } 3 \mathrm{~d}\end{array}$ & $\begin{array}{l}\text { Interrater: Pearson } r=.87 ; r^{2}=.77 \\
\text { Intrarater: Pearson } r=.98 ; r^{2}=.97\end{array}$ \\
\hline $\begin{array}{l}\text { DPAQ; Gremeaux } \\
\text { et } \mathrm{al}^{50}\end{array}$ & Interrater & $\begin{array}{l}\text { PAS in points out of } 30 \text { where } 0-10=\text { highly } \\
\text { sedentary and } 21-30=\text { highly active }\end{array}$ & $10 \mathrm{~d}$ & $\begin{array}{l}\text { Cohen } k \text { for interrater reliability: cardiac } \\
\text { rehabilitation group }=0.27 \text {, } \\
\text { Noncardiac rehabilitation group }=0.68\end{array}$ \\
\hline $\begin{array}{l}\text { PAS; Mezzani } \\
\text { et } \mathrm{al}^{54}\end{array}$ & $\begin{array}{l}\text { Test-retest } \\
\text { Interrater }\end{array}$ & $\begin{array}{l}\text { Activity score based upon intensity (MET) and } \\
\text { frequency }\end{array}$ & $15 \mathrm{~d}$ & $\begin{array}{l}\text { Test-retest: } \mathrm{ICC}=.97 \\
\text { Interrater: } \mathrm{ICC}=.98\end{array}$ \\
\hline $\begin{array}{l}\text { PASE; } \\
\text { Allison et al } \\
55\end{array}$ & Test-retest & PASE score & $\begin{array}{l}\text { Within 2- } \\
3 \mathrm{wk}\end{array}$ & Pearson $r=.72$ \\
\hline
\end{tabular}

Abbreviations: DAQIHF, Daily Activity Questionnaire in Heart Failure Scale; DEE, Daily Energy Expenditure; DPAQ, Dijon Physical Activity Questionnaire; ICC = intraclass correlation coefficient; MET = metabolic equivalent or task; MobilePAL, Mobile Phone Physical Activity Logger; PAS, Physical Activity Score; PASE, Physical Activity Scale for the Elderly; SWISSPAQ, SWISS Physical Activity Questionnaire; TAM1, Total Activity Measure 1; TAM2, Total Activity Measure 2.

The subjective DPA measures, which estimate EE per kilocalories and which were compared against accelerometry, include the SWISSPAQ, ${ }^{38}$ TAM1, ${ }^{44}$ TAM2, ${ }^{44}$ and MobilePAL. ${ }^{46}$ Despite moderate to high reliability, the SWISSPAQ, TAM1, TAM2, and MobilePAL demonstrated overall low to moderate validity $(r=-.086 \text { to } .45)^{38,44,46}$ and tended to overestimate activity. These results related to validity for all 4 instruments have yet to be replicated, so it is not yet known whether these results are spurious or whether these questionnaires have significant limitations. For example, the TAM1, TAM2, and SWISSPAQ ask respondents to estimate the typical or average activity, rather than report on the actual activity measured in a specific timeframe.

It is interesting to note that one of the most common reference standards used for validating DPA questionnaires was peak oxygen uptake. The theoretical basis for this choice of reference standard is the relationship between DPA and peak oxygen uptake. Individuals with greater activity levels typically have a greater peak oxygen uptake. ${ }^{63-65}$ Therefore, some studies selected peak oxygen uptake as a proxy objective measurement of activity level. The correlation coefficients of .30 to .71 with peak oxygen uptake $^{36,37,48,53,54}$ were greater than the low to moderate coefficients of .09 to .45 reported for the instruments that were compared against accelerometry. ${ }^{38,44,46}$ Similarly, other reference standards, including anthropometric measurements, exercise duration, and the 6-minute walk test, also had stronger correlation coefficients than accelerometry. ${ }^{36,37,49,57}$ However, the use of reference standards that are not direct measures of DPA are not recommended. ${ }^{59-61,66,67}$
Although the IPAQ was the most frequently studied questionnaire (6 studies), ${ }^{42,43,46,47,49,56}$ only one study compared it against accelerometry $(r=.20) .{ }^{42}$ Correlation coefficients with other reference standards were generally low to moderate. The strongest relationship was noted between the IPAQ activity category and pedometer-based steps per day $(r=.54),{ }^{56}$ which is consistent with previous research on the IPAQ in other populations. ${ }^{68}$ Despite the relatively low correlations between the IPAQ and objective DPA measures, it was used as a reference standard for validating the Mobile PAL ${ }^{46}$ and Acti'MET. ${ }^{49}$ None of the included studies assessed the IPAQ's reliability.

It should be noted that a recent study of a subjective measure of DPA in individuals undergoing cardiac rehabilitation examined the Past-day Adults' Sedentary Time (PAST) questionnaire. ${ }^{69}$ This study was not included in this review due to publication after our literature search was completed. The PAST measures the minutes of sedentary time in the past day and, similar to the other questionnaires included in this review, demonstrated low correlations with triaxial accelerometry. However, the authors observed improved overall agreement between the PAST and accelerometry over a 12-month period. The authors concluded that participants become better at estimating sedentary behavior and that the PAST may be useful for the measurement of changes in sedentary behavior at a group level following cardiac rehabilitation.

Similar to previous reviews, ${ }^{29,30,32}$ the authors of this review are unable to recommend a single subjective DPA measure for use with individuals with cardiovascular disease. However, this review is the most comprehensive to date with regard to search strategy, 
study populations, and recommendations and was focused solely on subjective DPA measures. Subjective DPA measures varied greatly with regard to the PA construct measured, length of recall, time/method of administration, and unit of measurement (Table 3). Test-retest reliability was generally good across the instruments in which it was studied, and therefore, these measures could be useful in identifying change in DPA. Unfortunately, relatively few questionnaires measuring METs, EE per kilocalories, or minutes of MVPA per week were compared against optimal reference standards of calorimetry, accelerometry, or DPA diaries. Of the questionnaires measuring METs, EE per kilocalories, or minutes of MVPA per week that were compared against the optimal reference standards of calorimetry, accelerometry, or DPA diaries (SWISSPAQ, ${ }^{38}$ TAM1/TAM2, ${ }^{44}$ and MobilePAL. ${ }^{46}$ ), the greatest differences were the recall period (ranging from less than $1 \mathrm{~d}$ to $2 \mathrm{mo}$ ) and construct measured/measurement units (MET hours of DPA vs minutes spent in different activity categories vs ratio of total daily $\mathrm{EE}$ to total resting EE). Therefore, the selection of a subjective measure of DPA would depend upon specific needs of the researcher or clinician in regard to the desired recall period (less than a day to $2 \mathrm{mo}$ ) and the construct measured (MET hours of DPA vs minutes spent in different activity categories vs ratio of total daily EE to total resting EE).

Future research on subjective DPA measures is clearly needed. The majority of the studies reviewed generally lacked high methodological rigor, and reliability testing was uncommon. Future studies should select reference standards most directly related to the DPA construct being measured and should seek to replicate results or prior studies.

This review was limited to peer-reviewed, English-only, fulltext articles. In addition, this review included studies reporting on relationships between subjective DPA measures and reference standards, even when the primary objective of the included study was not to establish the validity of a given questionnaire. Finally, the conclusions of this review are limited to studies conducted in individuals with heart disease, and therefore, validity/reliability for a given instrument in other populations may be different.

\section{Conclusions}

Of the 19 questionnaires identified in this review, only the SWISS Physical Activity Questionnaire, Total Activity Measure 1 and 2, and Mobile Physical Activity Logger 1) provide an estimate of energy expenditure, metabolic equivalents, or minutes of DPA, 2) have established test-retest reliability, and 3) have been validated using accelerometry or a DPA diary. ${ }^{38,44,46}$ Test-retest reliability was good to excellent; however, the validity coefficients were moderate at best. Depending on the clinician or researcher needs, the selection of a subjective measure of DPA would depend on the desired recall period (less than $1 \mathrm{~d}$ to $2 \mathrm{mo}$ ) and the construct measured (MET hours of DPA vs minutes spent in different activity categories vs ratio of total daily EE to total resting EE).

\section{Acknowledgments}

The authors are grateful to Betsy Williams, reference librarian at Grand Valley State University, for her assistance with the design and execution of the database searches. There were no sources of funding for this work. All authors have no disclosures of funding or any potential conflicts of interest.

\section{References}

1. Izawa KP, Watanabe S, Oka K, et al. Usefulness of step counts to predict mortality in Japanese patients with heart failure. Am J Cardiol. 2013;111(12):1767-1771. PubMed ID: 23540653 doi:10.1016/j. amjcard.2013.02.034

2. Shoemaker MJ, Curtis AB, Vangsnes E, Dickinson MG, Paul R. Analysis of daily activity data from implanted cardiac defibrillators: the minimum clinically important difference and relationship to mortality/life expectancy. World J Cardiovasc Dis. 2012;2(3): 129-135. doi:10.4236/wjcd.2012.23021

3. Shoemaker MJ, Curtis AB, Paul R, Dickinson MG, Polso A, Kaushik S. Longitudinal daily activity patterns in individuals with heart failure: derivation and association with prognosis and clinical characteristics. Cardiopulm Phys Ther J. 2013;24(4):25-33. doi:10.1097/ 01823246-201324040-00005

4. Walsh JT, Charlesworth A, Andrews R, Hawkins M, Cowley AJ. Relation of daily activity levels in patients with chronic heart failure to long-term prognosis. Am J Cardiol. 1997;79(10):1364-1369. doi: 10.1016/S0002-9149(97)00141-0

5. Doukky R, Mangla A, Ibrahim Z, et al. Impact of physical inactivity on mortality in patients with heart failure. Am J Cardiol. 2016;117(7): 1135-1143. PubMed ID: 26853954 doi:10.1016/j.amjcard.2015. 12.060

6. Bouchard C, Blair SN, Katzmarzyk PT. Less sitting, more physical activity, or higher fitness? Mayo Clin Proc. 2015;90(11):1533-1540. PubMed ID: 26422244 doi:10.1016/j.mayocp.2015.08.005

7. Paffenbarger RS, Kampert JB, Lee IM, et al. Changes in physical activity and other lifeway patterns influencing longevity. Med Sci Sports Exerc. 1994;26(7):857-865.

8. Yavari M, Haykowsky MJF, Savu A, Kaul P, Dyck JRB, Haennel RG. Volume and patterns of physical activity across the health and heart failure continuum. Can J Cardiol. 2017;33(11):1465-1471. PubMed ID: 28985961 doi:10.1016/j.cjca.2017.07.005

9. Loprinzi PD. Implications of light-intensity physical activity in improving health-related quality of life among congestive heart failure patients. Int J Cardiol. 2016;212:16-17. PubMed ID: 27015644 doi:10.1016/j.ijcard.2016.03.015

10. Tung HH, Jan MS, Lin CY, Chen SC, Huang HC. Mediating role of daily physical activity on quality of life in patients with heart failure. J Cardiovasc Nurs. 2012;27(1):16-23. PubMed ID: 21558866 doi: 10.1097/JCN.0b013e318211284b

11. Pearson MJ, Smart NA. Exercise therapy and autonomic function in heart failure patients: a systematic review and meta-analysis. Heart Fail Rev. 2018;23(1):91-108. PubMed ID: 29185161 doi:10.1007/ s10741-017-9662-z

12. Saavedra MJ, Romero F, Roa J, Rodriguez-Nunez I. Exercise training to reduce sympathetic nerve activity in heart failure patient. A systematic review and meta-analysis. Braz J Phys Ther. 2018;22(2): 97-104. PubMed ID: 28733092 doi:10.1016/j.bjpt.2017.06.014

13. Cui J, Muller MD, Blaha C, Kunselman AR, Sinoway LI. Seasonal variation in muscle sympathetic nerve activity. Physiol Rep. 2015; 3(8):e12492. doi:10.14814/phy2.12492

14. Levin R, Katz M, Saldiva P, et al. Increased hospitalization for decompensated heart failure and acute myocardial infarction during mild winters: a seven-year experience in the public health system of the largest city in Latin America. PLoS One. 2018;13(1):e0190733. PubMed ID: 29300764 doi:10.1371/journal.pone.0190733

15. Kaneko H, Suzuki S, Goto M, et al. Presentations and outcomes of patients with acute decompensated heart failure admitted in the winter season. J Cardiol. 2014;64(6):470-475. PubMed ID: 24802170 doi:10.1016/j.jjcc.2014.03.004 
16. Klompstra L, Jaarsma T, Stromberg A, et al. Seasonal variation in physical activity in patients with heart failure. Heart Lung. 2019; 48(5):381-385. PubMed ID: 31122692 doi:10.1016/j.hrtlng.2019. 04.003

17. Lahti-Koski M, Pietinen P, Heliovaara M, Vartiainen E. Associations of body mass index and obesity with physical activity, food choices, alcohol intake, and smoking in the 1982-1997 FINRISK Studies. Am J Clin Nutr. 2002;75(5):809-817. PubMed ID: 11976153 doi:10. 1093/ajcn/75.5.809

18. Kriska AM, Hanson RL, Bennett PH, et al. Physical activity, obesity, and the incidence of type 2 diabetes in a high-risk population. Am J Epidemiol. 2003;158(7):669-675. PubMed ID: 14507603 doi:10. 1093/aje/kwg191

19. Rennie KL, McCarthy N, Yazdgerdi S, Marmot M, Brunner E. Association of the metabolic syndrome with both vigorous and moderate physical activity. Int J Epidemiol. 2003;32(4):600-606. PubMed ID: 12913036 doi:10.1093/ije/dyg179

20. Kaminsky LA, Brubaker PH, Guazzi M, et al. Assessing physical activity as a core component in cardiac rehabilitation: a position statement of the american association of cardiovascular and pulmonary rehabilitation. J Cardiopulm Rehabil Prev. 2016;36(4):217-219.

21. U.S. Department of Health and Human Services, Physical Guidelines for Americans. 2nd ed. Washington, DC: U.S. Department of Health and Human Services; 2018.

22. Dohrn IM, Kwak L, Oja P, Sjostrom M, Hagstromer M. Replacing sedentary time with physical activity: a 15-year follow-up of mortality in a national cohort. Clin Epidemiol. 2018;10:179-186. PubMed ID: 29416378 doi:10.2147/CLEP.S151613

23. Diaz KM, Howard VJ, Hutto B, et al. Patterns of sedentary behavior and mortality in U.S. middle-aged and older adults: a national cohort study. Ann Intern Med. 2017;167(7):465-475. PubMed ID: 28892811 doi:10.7326/M17-0212

24. Bauman A, Phongsaval P, Schoeppe S, Owen N. Physical activity measurement a primer for health population. Promot Educ. 2006; 13(2):92-103. doi:10.1177/10253823060130020103

25. Lim S, Wyker B, Bartley K, Eisenhower D. Measurement error of self-reported physical activity levels in New York City: assessment and correction. Am J Epidemiol. 2015;181(9):648-655. PubMed ID: 25855646 doi:10.1093/aje/kwu470

26. Urda JL, Larouere B, Verba SD, Lynn JS. Comparison of subjective and objective measures of office workers' sedentary time. Prev Med Rep. 2017;8:163-168. PubMed ID: 29062680 doi:10.1016/j.pmedr. 2017.10.004

27. Warren JM, Ekelund U, Besson H, et al. Assessment of physical activity - a review of methodologies with reference to epidemiological research: a report of the exercise physiology section of the European Association of Cardiovascular Prevention and Rehabilitation. Eur J Cardiovasc Prev Rehabil. 2010;17(2):127-139. PubMed ID: 20215971 doi:10.1097/HJR.0b013e32832ed875

28. Strath SJ, Kaminsky LA, Ainsworth BE, et al. Guide to the assessment of physical activity: clinical and research applications: a scientific statement from the American Heart Association. Circulation. 2013;128(20):2259-2279. PubMed ID: 24126387 doi:10.1161/01. cir.0000435708.67487.da

29. Le Grande MR, Elliot PC, Worcester MU, Murphy BM, Goble AJ. An evaluation of self-report physical activity instruments used in studies involving cardiac patients. J Cardiopulm Rehabil Prev. 2008; 28(6):358-369. PubMed ID: 19008689 doi:10.1097/HCR.0b013e $31818 \mathrm{c} 3 \mathrm{~d} 90$

30. Ahlarbi M, Bauman A, Neubeck L, Gallagher R. Measuring overall physical activity for cardiac rehabilitation participants: a review of the literature. Heart Lung Circ. 2017;26(1):1008-1025.
31. Savage PD, Toth MJ, Ades PA. A re-examination of the metabolic equivalent concept in individuals with coronary heart disease. $J$ Cardiopulm Rehabil Prev. 2007;27(3):143-148. PubMed ID: 17558194 doi:10.1097/01.HCR.0000270693.16882.d9

32. Panguntalan J, Gregoski M. An integrative review of self-report instruments for measuring physical activity among African American women: a coronary heart disease perspective. J Black Stud. 2015; 46(2):218-236.

33. Lucas NP, Macaskill P, Irwig L, Bogduk N. The development of a quality appraisal tool for studies of diagnostic reliability (QAREL). J Clin Epidemiol. 2010;63(8):854-861. PubMed ID: 20056381 doi: 10.1016/j.jclinepi.2009.10.002

34. Gore S, Goldberg A, Huang MH, Shoemaker M, Blackwood J. Development and validation of a quality appraisal tool for validity studies (QAVALS). Physiother Theory Pract. 2019;27:1-9. PubMed ID: 31246154 doi:10.1080/09593985.2019.1636435

35. Von Elm E, Altman D, Egger, M, Pocock S, Gøtzsche P, Vandenbroucke J. The strengthening the reporting of observational studies in epidemiology (STROBE) statement: guidelines for reporting observational studies. J Clin Epidemol. 2008;61(4):344-349. doi:10.1016/ j.jclinepi.2007.11.008

36. Garet M, Barthelemy JC, Degache F, et al. A questionnaire-based assessment of daily physical activity in heart failure. Eur J Heart Fail. 2004;6(5):577-584. PubMed ID: 15302005 doi:10.1016/j.ejheart. 2003.11.022

37. Garet M, Degache F, Costes F, et al. DAQIHF: methodology and validation of a daily activity questionnaire in heart failure. Med Sci Sports Exerc. 2004;36(8):1275-1282. PubMed ID: 15292732 doi:10. 1249/01.MSS.0000135776.09613.0D

38. Bahler C, Bjarnason-Wehrens B, Schmid JP, Saner H. SWISSPAQ: Validation of a new physical activity questionnaire in cardiac rehabilitation patients. Swiss Med Wkly. 2013;143:w13752. PubMed ID: 23519959

39. Tayor-Piliae RE, Haskell WL, Iribarren C, et al. Clinical utility of the stanford brief activity survey in mean and women with early-onset coronary artery disease. J Cardiopulm Rehabil Prev. 2007;27(4): 227-232. PubMed ID: 17667019 doi:10.1097/01.HCR.0000281768. 97899.bb

40. Chien HC, Chen HM, Garet M, Wang RH. Predictors of physical activity in patients with heart failure: a questionnaire study. $J$ Cardiovac Nurs. 2014;29(4):324-331. PubMed ID: 23416940 doi:10. 1097/JCN.0b013e31828568d6

41. Gruner C, Alig F, Muntwyler J. Validity of self-reported exerciseinduced sweating as a measure of physical activity among patients with coronary artery disease. Swiss Med Wkly. 2002;132(43-44): 629-632. PubMed ID: 12587047

42. Larsson L, Johansson B, Wadell K, Thilen U, Sandberg C. Adults with congenital heart disease overestimate their physical activity level. Int J Cardiol Heart Vasc. 2018;22:13-17. PubMed ID: 30480085 doi:10.1016/j.ijcha.2018.11.005

43. Prince SA, Reid RD, Reed JL. Comparison of self-reported and objectively measured levels of sitting and physical activity and associations with markers of health in cardiac rehabilitation patients. Eur J Prev Cardiol. 2019;26(6):653-656. doi:10.1177/20474873 18806357

44. Orrell A, Doherty P, Miles J, Lewin R. Development and validation of a very brief questionnaire measure of physical activity in adults with coronary heart disease. Eur J Cardiovasc Prev Rehabil. 2007;14(5): 615-623. PubMed ID: 17925619 doi:10.1097/HJR.0b013e3280 ecfd56

45. Orrell A, Doherty P, Coulton S, Miles J, Stamatakis E, Lewin R. Failure to validate the health survey for England physical activity 
module in a cardiac population. Health Policy. 2007;84(2-3): 262-268. PubMed ID: 17498835 doi:10.1016/j.healthpol.2007. 03.004

46. Pfaeffli L, Maddison R, Jiang Y, Dalleck L, Lof M. Measuring physical activity in a cardiac rehabilitation population using a smartphone-based questionnaire. J Med Internet Res. 2013;15(3): e61. PubMed ID: 23524251 doi:10.2196/jmir.2419

47. Muller J, Amberger T, Berg A, et al. Physical activity in adults with congenital heart disease and associations with functional outcomes. Heart. 2017;103(14):1117-1121. PubMed ID: 28274955

48. Nowak Z, Plewa M, Skowron M, Markiewicz A, Kucio C, Osiadlo G. Paffenbarger physical activity questionnaire as an additional tool in clinical assessment of patients with coronary artery disease treated with angioplasty. Kardiol Pol. 2012;68(1):32-39.

49. Satge J, Guiraid T, Carette P, Granger R, Labrunee M. Reliability and concurrent validity of the Acti'MET® calculator: a new tool to assess physical activity in cardiac rehabilitation. Ann Phys Rehabil Med. 2015;58(3):157-160 PubMed ID: 25958005 doi:10.1016/j.rehab. 2015.01.004

50. Gremeaux V, Lemoine Y, Fargeot A, et al. The Dijon Physical Activity Score: reproducibility and correlations with physical fitness in patients with coronary artery disease. Ann Readapt Med Phys. 2008;51(5):366-378. PubMed ID: 8599146 doi:10.1016/j.annrmp. 2008.05.003

51. Sundal Holen M, Een R, Mildestvedt T, Eide GE, Meland E. Two valid measures of self-rated physical activity and capacity. Open Cardiovasc Med J. 2012;6(1):156-162. PubMed ID: 23346259 doi: 10.2174/1874192401206010156

52. Guiraud T, Granger R, Bousquet M, Gremeaux V. Validity of a questionnaire to assess the physical activity level in coronary artery disease patients. Int J Rehabil Res. 2012;35(3):270-274. PubMed ID: 22584380 doi:10.1097/MRR.0b013e3283531023

53. Myers J, Gullestad L, Bellin D, Ross H, Vagelos R, Fowler M. Physical activity patterns and exercise performance in cardiac transplant recipients. J Cardiopulm Rehabil. 2003;23(2):100-106. PubMed ID: 12668931 doi:10.1097/00008483-200303000-00006

54. Mezzani A, Corra U, Baroffio C, Bosimini E, Giannuzzi P. Habitual activities and peak aerobic capacity in patients with asymptomatic and symptomatic left ventricular dysfunction. Chest. 2000;117(5): 1291-1299. PubMed ID: 10807813 doi:10.1378/chest.117.5.1291

55. Allison MJ, Keller C, Hutchinson PL. Selection of an instrument to measure the physical activity of elderly people in rural areas. Rehabil Nurs. 1998;23(6):309-314. PubMed ID: 10223034 doi:10.1002/j. 2048-7940.1998.tb01811.x

56. Borland M, Rosenkvist A, Cider A. A group-based exercise program did not improve physical activity in patients with chronic heart failure and comorbidity: a randomized controlled trial. J Rehabil Med. 2014;46(5):461-467. PubMed ID: 24691771 doi:10.2340/ $16501977-1794$
57. Oka RK, DeMarco T, Haskell WL. Perceptions of physical fitness in patients with heart failure. Prog Cardiovasc Nurs. 1999;14(3): 97-102. PubMed ID: 10549047

58. Ribeiro-Samora GA, Pereira DA, Vieira OA, et al. Using the human activity profile to assess functional performance in heart failure. J Cardiopulm Rehabil Prev. 2016;36(3):180-185. PubMed ID: 26784733 doi:10.1097/HCR.0000000000000162

59. Shephard R, Vuillemin A. Limits to the measurement of habitual physical activity by questionnaires. Br J Sports Med. 2003;37(3): 197-206. PubMed ID: 12782543 doi:10.1136/bjsm.37.3.197

60. Vanhees L, Lefevre J, Philippaerts R, et al. How to assess physical activity? How to assess physical fitness? Eur J Cardiovasc Prev Rehabil. 2005;12(2):102-114. PubMed ID: 15785295 doi:10.1097/ 01.hjr.0000161551.73095.9c

61. Calabro M, Kim Y, Franke W, Stewart J, Welk G. Objective and subjective measurement of energy expenditure in older adults: a doubly labeled water study. Eur J Clin Nutr. 2015;69(7):850-855. PubMed ID: 25351651 doi:10.1038/ejen.2014.241

62. Rush E, Valencia M, Plank L. Validation of a 7-day physical activity diary against doubly-labelled water. Ann Hum Biol. 2008;35(4): 416-421. PubMed ID: 18608111 doi:10.1080/03014460802089825

63. Mundwiler J, Schupbach U, Dieterle T, et al. Association of occupational and leisure-time physical activity with aerobic capacity in a working population. PLoS One. 2017;12(1):e0168683. PubMed ID: 28045939 doi:10.1371/journal.pone.0168683

64. Morie M, Reid KF, Miciek R, et al. Habitual physical activity levels are associated with performance in measures of physical function and mobility in older men. $J$ Am Geriatr Soc. 2010;58(9):1727-1733. PubMed ID: 20738436 doi:10.1111/j.1532-5415.2010.03012.x

65. Knapik J, Zoltick J, Rottner C, et al. Relationships between selfreported physical activity and physical fitness in active men. Am J Prev Med. 1993;9(4):203-208. PubMed ID: 8398219 doi:10.1016/ S0749-3797(18)30716-5

66. Jacobs DR Jr, Ainsworth BE, Hartman TJ, Leon AS. A simultaneous evaluation of 10 commonly used physical activity questionnaires. Med Sci Sports Exerc. 1993;25(1):81-91. PubMed ID: 8423759 doi: 10.1249/00005768-199301000-00012

67. Wareham NJ, Rennie KL. The assessment of physical activity in individuals and populations: why try to be more precise about how physical activity is assessed? Int J Obes Relat Metab Disord. 1998; 22(suppl 2):S30-S38. PubMed ID: 9778094

68. Lee PH, Macfarlane DJ, Lam TH, Stewart SM. Validity of the international physical activity questionnaire short form (IPAQ-SF): a systematic review. Int J Behav Nutr Phys Act. 2011;8(1):115. PubMed ID: 22018588 doi:10.1186/1479-5868-8-115

69. Freene N, McManus M, Mair T, Tan R, Clark B, Davey R. Validity of the past-day Adults' Sedentary Time Questionnaire in a cardiac rehabilitation population. J Cardiopulm Rehabil Prev. 2020;40(5):325-329. PubMed ID: 31972632 doi:10.1097/HCR.0000000000000488 


\section{Erratum: Shoemaker et al. (2021)}

In the original publication of this article, the list of databases searched was inaccurately listed as PubMed, CINAHL, MEDLINE, and ProQuest. The list of databases have been updated to correctly read PubMed, CINAHL Complete, PsycInfo, and Web of Science Core Collection. The authors apologize for this error. 